\title{
Estilos de aprendizaje y rendimiento académico de universitarios de Educación Física chilenos Learning styles and academic performance of Chilean Physical Education university students *Jaime Serra-Olivares, *Carlos Leonel Muñoz Valverde, **Consuelo CejudoArmero, **Pedro Gil Madrona *Universidad Católica de Temuco (Chile), **Universidad de Castilla-La Mancha (España)
}

\begin{abstract}
Resumen. Se analizó la relación entre los estilos de aprendizaje y el rendimiento académico de los estudiantes universitarios de Educación Física de la Universidad Católica de Temuco en Chile 2015/16 (n = 122). Los participantes completaron el cuestionario CHAEA-36 y se analizaron los datos en función del curso, la edad y el género. Los hallazgos demostraron la predominancia, principalmente, de los estilos de aprendizaje combinado (56\%) y activo (21\%). No se observó relación significativa entre los estilos de aprendizaje y el curso $\left(x^{2}=14.324 ; p=.575\right)$, y tampoco la edad $\left(x^{2}=14.324\right.$; $p=.575)$. Sin embargo, el análisis inferencial demostró una relación significativa entre los estilos de aprendizaje y el género ( $x^{2}=11.257$; $\left.p=.024\right)$. Por último, los estudiantes con un estilo de aprendizaje activo demostraron un rendimiento académico significativamente inferior que sus compañeros con estilos de aprendizaje pragmático $(F=9.517 ; p=.011)$ y combinado $(F=14.625 ; p=.013)$. No se observaron diferencias significativas en el resto de comparaciones. Estos hallazgos determinan la importancia de atender a los procesos de formación inicial docente desde una perspectiva flexible, adaptada al contexto sociocultural y educativo de los aprendices. En este sentido, se necesitan nuevos estudios de investigación que analicen la relación entre los estilos de aprendizaje y el rendimiento académico, principalmente desde la perspectiva de género.
\end{abstract}

Palabras clave: Estilos de Aprendizaje, Formación Inicial Docente, Educación Superior, Rendimiento académico.

Abstract: The relationship between learning styles and academic performance of physical education students at the Temuco Catholic University 2015/16 was analysed $(n=122)$. Participants completed the CHAEA-36 questionnaire and data were analysed by academic year, age and gender. The findings showed a predominance of a combined style of learning (56\%) and the active learning style (21\%). No significant relationship between learning styles and academic year $\left(x^{2}=14.324 ; p=.575\right)$ nor age $\left(x^{2}=14.324 ; p=.575\right)$ were observed. However, the inferential analysis showed a significant relationship between learning styles and gender $\left(x^{2}=11.257 ; p=.024\right)$. Finally, students with an active learning style demonstrated significantly lower academic performance than their peers using pragmatic $(F=9.517 ; p=.011)$ and combined $(F=14.625 ; p=.013)$ learning styles. No other significant differences were observed. These findings show the importance of approaching teachers basic training from a flexible perspective, adapting it to learners' cultural and educational context. In this sense, new research studies that analyse the relationship between learning styles and academic performance, mainly from the gender perspective, are needed.

Keywords: Learning Styles, Initial Teacher Training, Higher Education, Academic Performance.

\section{Introducción}

El proceso de formación universitaria supone un constructo complejo para los diferentes actores del sistema educativo. En el caso de directivos y profesores, cómo adaptar el currículum a las necesidades y características de los estudiantes, sigue siendo un determinante fundamental en la construcción de procesos de enseñanza-aprendizaje de calidad (Madrid-López, Prieto-Ayuso, Samalot-Rivera, \& Gil-Madrona, 2016). Los estudiantes analizan y estructuran la información de diferentes formas para desarrollar el aprendizaje (Acevedo, Cavadla, \& Avis, 2015). Así, los estilos de aprendizaje han cobrado especial importancia en la literatura científica actual relacionada con la formación inicial docente (Gil-Madrona et al., 2007; González., Valenzuela, \& González, 2015; Ortiz \& Canto, 2013) y dentro de la actividad física y el deporte (Grupera, 2007; Zamorano-García, Gil-Madrona, Prieto-Ayuso \& Zamorano-García (201x). Los diferentes estilos de aprendizaje, junto a otras variables como las expectativas del alumno, el entorno personal, las habilidades sociales o el autoconcepto, se relacionan estrechamente con el rendimiento académico (Edel, 2003; Garbanzo, 2007; Margalef, 2014; Monroy \& Hernández, 2014; Núñez \& Reyes, 2014; Vélez \& Roa, 2005). Por estos motivos, se percibe la necesidad de conocer de qué manera aprenden los universitarios, también en función de su edad y género (Delgado Noguera \& Zurita, 2003; Urval et al., 2014). Este conocimiento permitiría adaptar los procesos de formación y alcanzar mayores resultados de aprendizaje, resultados cuantificables (Catalán \& Santelices, 2014; Gómez et al., 2015; Ramírez \& Fuentes, 2013; Villalta, 2010).

Los estilos de aprendizaje pueden definirse como los diferentes mecanismos mediante los cuales la mente procesa y asimila información, influida por las percepciones, durante la adquisición de estructuras de conocimiento superior (Gil-Madrona, 2004 y Gil-Madrona et al., 2007). Se caracterizan por ser esquemas de comportamiento flexibles y cambiantes dependiendo, entre otros, del contexto del aprendiz, su

Fecha recepción: 01-03-16. Fecha de aceptación: 23-11-16

Ciria Margarita Salazar

grillosalazar@gmail.com experiencia, y las estrategias docentes en las que se ve inmerso(Bahamón et al., 2012; Isaza, 2014). Son varios los expertos, los que han contribuido a la construcción de un modelo explicativo sobre los estilos de aprendizaje, siendo el modelo de Honey y Mumdford el más aceptado en la actualidad. Este modelo admite diversas potencialidades de un aprendiz en las cuatro dimensiones-estilos principales del aprendizajeexperiencia (Maureira, 2015), permitiendo reconocer la predominancia del estilo de aprendizaje de un estudiante, y también sus características (Acevedo et al., 2015; Bahamón et al., 2013; García \& De León, 2014; González et al., 2015; Villalba, 2015):

- Cuando predomina un estilo de aprendizaje activo, los estudiantes se caracterizan por ser animadores, espontáneos, arriesgados, creativos, innovadores, y participativos. Tienen facilidad para trabajar en grupo, y disfrutan de las nuevas experiencias. Sin embargo, si bien se entusiasman con lo nuevo, prontamente se desencantan y necesitan buscar algo diferente.

- Cuando impera un estilo de aprendizaje reflexivo, los estudiantes son observadores, receptores y analíticos. Son concienzudos en su quehacer, e intentan comprender los significados y describir procesos del aprendizaje. Se caracterizan por ser detallistas y observan las situaciones desde diferentes perspectivas, reflexionando antes de actuar. Poseen dificultades para aprender cuando no tienen datos suficientes para procesar información, realizan trabajos superficiales, o no han planificado.

- Por su parte, los estudiantes en los que predomina un estilo de aprendizaje teórico, son disciplinados, razonan y son prevalece. Emplean con frecuencia la abstracción para conformar conclusiones, y se aproximan al nuevo aprendizaje de forma objetiva, crítica, estructurada y planificada.

- Por último, los estudiantes que poseen un estilo de aprendizaje más pragmático suelen ser estudiantes que logran aprendizajes más profundos, al tener la posibilidad de aplicar de forma inmediata lo aprendido. Experimentan y elaboran planes de acción con resultados evidentes, aportando indicaciones pertinentes durante el trabajo grupo. Son realistas, decididos y concretos, aunque poseen dificultades cuando el contenido no tiene aplicación práctica en la realidad contextual

En general, los hallazgos observados en la literatura indican la predominancia de un estilo de aprendizaje más reflexivo en estudiantes universitarios (Acevedo et al., 2015; Camarero et al., 2000; Coloma et 
al., 2008; Esguerra \& Guerrero, 2009; Gil-Madrona etal., 2007; González et al., 2015; Loret de Mola, 2008; Ortiz \& Canto, 2013; Salas, 2014; Villalba, 2015). No obstante, existen estudios en los que se ha observado la prevalencia de otros estilos, caracterizados por su vinculación con procesos de enseñanza más tradicional en educación superior. Por ejemplo, Santizo et al. (2008) comprobaron la predominancia de un estilo de aprendizaje más teórico en estudiantes universitarios. Por el contrario, durante los últimos años se ha observado la predominancia de estilos más propios de las nuevas corrientes de enseñanza-aprendizaje, como el pragmático, en estudiantes de diferentes carreras (Izasa, 2014), o la combinación de estilos, que son sugeridos como estilos de mayor calidad y transferencia (Bahamón et al., 2013; Cordero et al., 2015).

Los estilos de aprendizaje también han sido analizados en función del semestre o curso académico en el que se encuentran los estudiantes (Gil-Madrona et al., 2007; Villalba, 2015) y su relación con las TIC (De Moya, Hernández, Hernández, \& Cózar, 2011). En este sentido, los hallazgos sugieren que durante el transcurso de una carrera universitaria los estilos de aprendizaje se modifican. Así, la predominancia o tendencia de ciertos estilos se vería alterada durante los últimos semestres y periodos de finalización. Este aspecto estaría relacionado no solamente con el tipo de metodologías desarrolladas por los docentes, sino también con la acomodación del aprendiz al/los nuevos contenidos y conocimientos a desarrollar, y con su habilidad para adaptarse al contexto en donde el entorno social es clave (Cocca, Mayorga-Vega, \& Viciana, 2013). En la misma línea, se ha analizado la influencia de la edad y el género sobre los estilos de aprendizaje de los estudiantes, aunque los hallazgos observados son dispares, y se necesitan más estudios que analicen la relación entre tales variables (Bahamón et al., 2013; GilMadrona et al., 2007; Villalba, 2015).

Finalmente, existe una línea de estudio de análisis de la relación entre los diferentes estilos de aprendizaje y el rendimiento académico, aunqueésta no ha sido desarrollada en profundidad(Wilkinson, Boohan, \& Stevenson, 2014). Se intuye que, gracias al conocimiento de tal relación, podría identificarse qué estilo/os sería preferible desarrollar para el logro de niveles de aprendizaje y rendimiento académico superior. Los resultados de algunos estudios sugieren que un estilo de aprendizaje más reflexivo, estaría relacionado con niveles de rendimiento académico superior (Esguerra \& Guerrero, 2009). Igualmente, parece ser que junto al reflexivo, un estilo de aprendizaje teórico también contribuiría al logro de niveles de rendimiento académico más altos (Ossa \& Lagos, 2013). Por último, el estilo de aprendizaje activo estaría relacionado con resultados de rendimiento académico inferiores, sugiriendo dificultades para el desarrollo de aprendizajes significativos (Camarero et al., 2000; Esguerro \& Guerrero, 2009).

Con todo lo anterior, conocer los diferentes estilos de aprendizaje de los estudiantes y su relación con el rendimiento académico, supondría una estrategia fundamental en la adecuación de los procesos curriculares en educación superior. En este sentido, como aproximación al tema en el contexto educativo chileno, el objetivo de este trabajo es identificar el/los estilos de aprendizaje predominantes de los estudiantes de Educación Física de la Universidad Católica de Temuco, y su relación con el rendimiento académico, en función del curso, la edad y el género. Las hipótesis asumidas corresponden a la observación de diferentes estilos de aprendizaje en función de estas variables. Igualmente, se espera observar relación entre los estilos de aprendizaje y el rendimiento académico, intuyendo que el estilo de aprendizaje reflexivo sería el más adecuado para el logro de resultados superiores.

\section{Método}

\section{Participantes y contexto}

Participaron todos los estudiantes de la carrera de Pedagogía en Educación Física de la Facultad deEducación de la Universidad Católica de Temuco en Chile matriculados el curso académico 2015/16 (n=122). No obstante debido a muerte experimental (por no contestar el cuestionario o debido a la pérdida de algunos datos), se seleccionó para análisis una muestra final de 95 estudiantes (69 chicos y 26 chicas, $22.8 \pm 0.3$ años de edad).

\section{Diseño y procedimiento}

Se desarrolló un estudio descriptivo, transversal, comparativo y correlacional (Martínez, 2007). Se obtuvo el consentimiento informado de la universidad participante y de los estudiantes. Se obtuvieron los datos del rendimiento académico en el final del curso 2015/16 (promedio de las calificaciones obtenidas en el total de asignaturas cursadas ese curso), en la Dirección General de Docencia de la universidad. Posteriormente, los estudiantes completaron el Cuestionario adaptado de Estilos de Aprendizaje CHAEA-36 (Maureira, 2015), adaptado de la versión original de Honey \& Alonso (Alonso, 1991).

Se encuestó a los estudiantes en su horario y lugar habitual de clase durante la última semana del calendario académico, en Enero de 2016. Las encuestas fueron realizadas en cinco sesiones/una sesión por día por el mismo investigador siguiendo el mismo protocolo. Primero, se entregaba el cuestionario, se explicaba cómo rellenarlo y se dejaban cinco minutos para aclarar dudas. Posteriormente, los estudiantes rellenaron el cuestionario y lo entregaron al investigador al finalizar el proceso.

\section{Variables y recogida de datos}

Se recopilaron datos respecto a los estilos de aprendizaje de los estudiantes, su rendimiento académico, curso, edad y género. El cuestionario CHAEA-36 ha sido utilizado en estudios anteriores. Dicho instrumento es una adaptación del cuestionario CHAEA original de Honey \& Alonso (Alonso, 1991), reducido de 80 a 36 ítems dicotómicos. Permite reducir el tiempo de respuesta, y posee valores de fiabilidad y validez satisfactorios (alfa de Cronbach, análisis factorial con rotación varimax y ANOVA), así como una alta correlación con el cuestionario original (Maueria, 2015). El instrumento, permite identificar la predominancia del estilo de aprendizaje del estudiante (activo, reflexivo, teórico o pragmático) respecto a otros, o la predominancia combinada de más de un estilo de aprendizaje (Tabla 1).

Tabla 1

Predominancia del estilo de aprendizaje del estudiante a partir de su puntuación en el Cuestionario de Estilos de Aprendizaje CHAEA-36 (adaptado de Maureira, 2013) \begin{tabular}{cccc} 
Cuestionario de Estilos de Aprendizaje & CHAEA-36 (adaptado de Maureira, 2013) \\
\hline Nivel Activo & Reflexivo Teórico & Pragmático
\end{tabular} Bajo $=5$ Activo $=6^{\text {Reflexivo }}=5$ Teórico $=5$ $\begin{array}{lllll}\text { Bajo } & =5 & =6 & =5 & =5 \\ \text { Medio } & 6-7 & 7-8 & 6-7 & 6-7\end{array}$

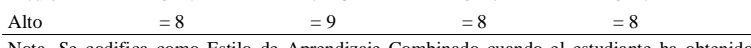
puntuación Nivel Alto en más de un estilo, o en su defecto, sin poseer Nivel Alto en ningún estilo, presenta Nivel Medio en más de un estilo.

\section{Análisis}

Se organizaron y clasificaron los datos en función del curso de la carrera de Educación Física $\left(1^{\circ}, 2^{\circ}, 3^{\circ}, 4^{\circ} 05^{\circ}\right.$ curso $)$, la edad, y el género. Se utilizaron las diferentes clasificaciones observadas en los estilos de aprendizaje predominantes (activo, reflexivo, teórico, pragmático, o combinado), con el propósito de analizar su relación con el rendimiento académico. Igualmente, la variable edad fue clasificada en tres grupos (18-20, 21-23 y 24-30) con fines de análisis. Se desarrolló el análisis descriptivo de los porcentajes de cada variable. Se realizó el análisis de la normalidad y la homogeneidad de las variables. Éstas cumplieron los supuestos necesarios para la utilización de pruebas paramétricas. Se utilizaron pruebas T Student para determinar si existían diferencias en el rendimiento académico en función del estilo de aprendizaje predominante de los estudiantes. Igualmente, se utilizó la prueba de Chi Cuadrado de Pearson para analizar la relación entre los estilos de aprendizaje, el curso y el género.

\section{Resultados}

\section{Predominancia de los Estilos de Aprendizaje}

Se observó la predominancia de un estilo de aprendizaje combinado en el 56\% de los estudiantes (Gráfico 1). Las combinaciones más representativas fueron Estilo Reflexivo-Teórico y Estilo Activo-TeóricoPragmático. El resto de estudiantes mostró la predominancia de los estilos Activo (21\%), Teórico (13\%) y Pragmático (4\%). 


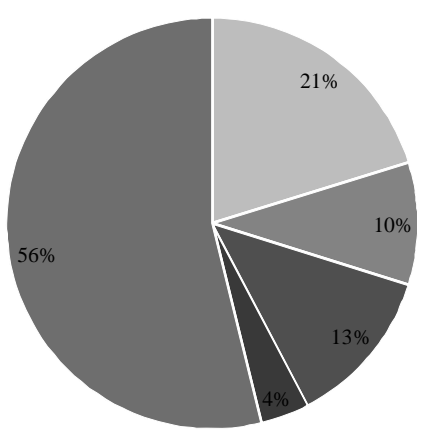

- Activo

- Reflexivo

- Teórico

- Pragmático

- Combinado

Gráfico 1. Predominancia de los estudiantes en los distintos tipos de estilos de aprendizaje.

Diferencias en los Estilos de Aprendizaje en función del curso

Los estudiantes mostraron la predominancia más baja de un estilo combinado en el $1^{\circ}$ y $4^{\circ}$ curso (50\%), y el más alto en $5^{\circ}$ (69\%). El porcentaje de estudiantes con predominancia en los estilos Activo y Teórico son similares a excepción del $3^{\circ}$ curso. En el $5^{\circ}$ curso se observó una ausencia de estudiantes con estilos Reflexivo y Pragmático (Gráfico 2). En cualquier caso, no se observó relación significativa entre el curso y el estilo de aprendizaje $\left(x^{2}=14.324 ; p=.575\right)$.

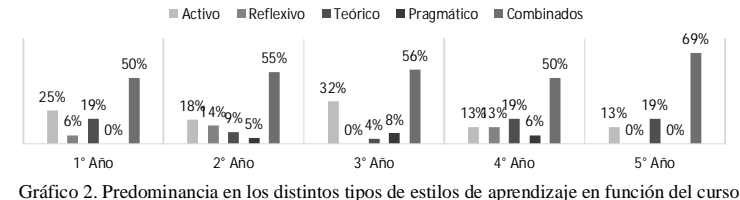

\section{Estilos de Aprendizaje y Género}

El género femenino mostró mayor predominancia que el masculino en el estilo de aprendizaje activo. El masculino, presentó una predominancia superior en la combinación de estilos. No se observaron diferencias de género en el resto de estilos de aprendizaje (Gráfico 3). El análisis inferencial determinó relación significativa entre el género y el estilo de aprendizaje de los estudiantes $\left(x^{2}=11.257 ; p=.024\right)$.

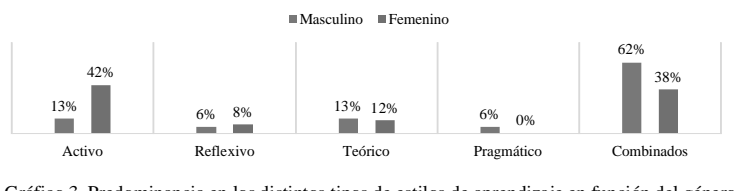

\section{Estilos de Aprendizaje y Edad}

El análisis descriptivo no desveló diferencias entre los grupos de edad en la predominancia de los estilos de aprendizaje. El porcentaje más alto se observó en el grupo de 18-20 años en la combinación de diferentes estilos (62\%) y en el estilo activo (29\%) (Gráfico 4). El análisis inferencial mostró que no existe relación significativa entre la edad y los estilos de aprendizaje $\left(x^{2}=14.324 ; p=.575\right)$.

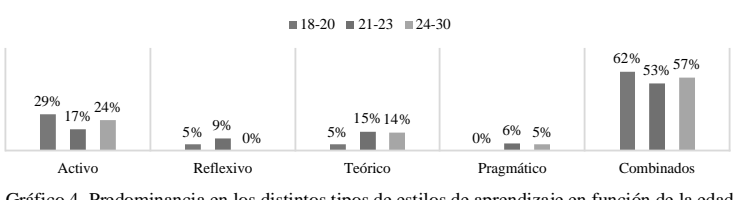

Estilos de Aprendizaje y Rendimiento Académico

Se observa que los estudiantes que presentan predominancia en los estilos de aprendizaje pragmático, combinado y teórico, presentan un rendimiento académico ligeramente superior que sus compañeros (Tabla2).

Los estudiantes con predominancia en el estilo de aprendizaje acti-
Tabla 2

Rendimiento académico en función del estilo de aprendizaje predominante

Estilo de Aprendizaie Rendimiento Académico

Activo

Reflexivo

Teórico

Pragmático

Combinado

$\frac{M}{39.65}$

39.65
48.50

48.50

56.25

$\underline{D T}$

23.87
21.04

17.36

5.32
14.89

vo mostraron un rendimiento académico significativamente inferior que sus compañeros con un estilo pragmático $(F=9.517 ; p=.011)$ y combinado $(F=14.625 ; p=.013)$. No se observaron diferencias significativas en el rendimiento académico al comparar los estudiantes con predominancia en un estilo de aprendizaje activo y reflexivo $(F=1.524$; $p=.40)$, y activo y teórico $(F=1.427 ; p=.157)$. No se observaron diferencias significativas en el rendimiento académico entre los estudiantes con predominancia en los estilos reflexivo y teórico ( $F=.052 ; p$ $=.778)$, reflexivo y pragmático $(F=1.967 ; p=.499)$ y reflexivo y combinado $(F=.837 ; p=.652)$. De igual modo, no se observaron diferencias entre los estilos teórico y pragmático $(F=2.414 ; p=.581)$ y teórico y combinado ( $F=.778 ; p=.941)$, ni entre pragmático y combinado $(F=1.005 ; p=.534)$.

\section{Discusión}

Los resultados demostraron que los estudiantes participantes en el estudio poseían diferentes estilos de aprendizaje, siendo el estilo combinado el de mayor predominancia. La edad y el curso no se relacionaron directamente con los estilos de aprendizaje. Por el contrario, el género demostró ser una variable significativamente relacionada. Finalmente, se observó que los estilos de aprendizaje estaban directamente relacionados con el rendimiento académico, siendo los estudiantes con un estilo activo los que poseían resultados significativamente inferiores al resto.A continuación, se discuten estos hallazgos en relación a los retos académicos y científicos de la formación inicial docente y la enseñanza superior, especialmente en el área de Educación Física y Deporte (Gómez-Mármol \& Valero, 2015; López Pastor et al., 2016; Zagalaz, Cachón, \& Lara, 2014).

\section{Predominancia de estilo de aprendizaje de los encuestados}

Los estudiantes mostraron diferentes estilos de aprendizaje, resaltando principalmente la presencia de un estilo combinado. Se observaron hasta diez combinaciones diferentes. Estos resultados son similares a otros estudios, como por ejemplo el de González et al. (2015), en el que se apreció un estilo de aprendizaje combinado en el $34 \%$ de los estudiantes. De igual modo, estos hallazgos están en la línea de los observados por Bahamón et al. (2013) en estudiantes universitarios de diferentes carreras, que mostraron predominancia en la combinación de estilos en el $48 \%$ de los casos.

La combinación de estilos presupone ciertas ventajas en el proceso educativo desde la perspectiva de los expertos en formación inicial docente. Permite la multimodalidad de desempeño cuando se desarrollan procesos de adquisición y aprendizaje de nuevos conocimientos (Bahamón et al., 2013). De este modo se pueden lograr objetivos relacionados con la formación profesional, dada la posibilidad de correspondencia con las estrategias de enseñanza implementadas (Cordero et al., 2015; González et al., 2015).

Otros estudios con estudiantes de Educación Física han mostrado resultados diferentes, determinando la predominancia de los estilos Reflexivo y Activo en los participantes (Gil et al., 2007; Salas, 2014), también en otras carreras universitarias (Acevedo et al., 2015; Camarero et al., 2000; Coloma et al., 2008; Esguerra \& Guerrero, 2009; Loret de Mola, 2008; Ortiz \& Canto, 2013; Villalba, 2015). De manera que sería común la observación de estudiantes con estos estilos en el entorno universitario. Este dato, junto al hecho de observar un $21 \%$ de estudiantes con predominancia en un estilo activo en este trabajo requiere especial atención, dada la relación con los procesos de enseñanza tradicionales (Santizo et al., 2008). Solamente observamos la predominancia por un estilo pragmático en uno de los estudios revisados, en estudiantes 
universitarios (Izasa, 2014), y un porcentaje muy bajo de estudiantes analizados aquí con un estilo pragmático. Estos resultados subrayan la necesidad de atender a los procesos de enseñanza empleados en la actualidad en los centros de educación superior, como el centro analizado en este trabajo (Urval et al., 2014).

\section{Estilos de aprendizaje y curso}

El hecho de observar un descenso en el porcentaje de estudiantes con un estilo de aprendizaje activo en los cursos superiores, junto con el aumento de los estudiantes con un estilo de aprendizaje combinado, sugiere la influencia del curso sobre la adaptación al a los procesos de enseñanza y aprendizaje, aunque no se observó relación significativa. En este sentido, es necesario un estudio longitudinal para comprobar la nueva hipótesis. Al parecer, el estudiante adaptaría su estilo de aprendizaje a medida que progresase en sus estudios, se viera influenciado por diferentes metodologías de enseñanza, y/o desarrollase procesos de maduración naturales(Villalba, 2015).

Estos resultados han sido observados también en otros trabajos como el de Gil-Madrona et al. (2007) o Villalba (2015), quienes observaron la modificación de los estilos de aprendizaje en los estudiantes a medida que avanzan en el devenir de sus respectivas carreras. Igualmente, esta idea conecta con la afirmación de Bahamón et al. (2013), quienes indican que los estilos de aprendizaje no son fijos, cambian según la situación de aprendizaje y experiencia del sujeto, modificándose y adaptándose a la vida universitaria y a las estrategias pedagógicas empleadas por los profesores. De este modo, la predominancia observada en este trabajo se debería a la influencia específica del contexto educativo concreto, en función del curso. En todo caso, son necesarios más trabajos de investigación, concretamente en esta muestra de estudio, para determinar la alta presencia de estudiantes con un estilo activo en el tercer curso analizado. Se intuye la presencia de otras variables como las metodologías empleadas por los profesores, el cambio de ciclo en el curriculum específico de la carrera, o la naturaleza de las prácticas en determinadas áreas de conocimiento como posibles influyentes de estos hallazgos.

\section{Estilos de aprendizajey género}

Los resultados mostraron la relación significativa entre el género y el estilo de aprendizaje. Este dato sugiere determinadas características que atender en el campo de la formación inicial docente para mejorar los procesos de enseñanza-aprendizaje de calidad (Carreiro da Costa, González Valeiro, \& González Villalobos, 2016). Se apreció un alto porcentaje de chicas con un estilo de aprendizaje activo, caracterizado por la espontaneidad, improvisación, asunción de riesgos y exploración; también representado por otras particularidades como la pérdida de interés en procesos de enseñanza-aprendizaje a largo plazo (Acevedo et al., 2015; Villalba, 2015). Este aspecto es de suma importancia, dadas las limitaciones del estilo activo para la construcción y adquisición de aprendizajes significativos. En el caso del género masculino, se sugiere que la combinación de diferentes estilos de aprendizaje, les permitiría utilizar diferentes estrategias o métodos para la adquisición de nuevos conocimientos.

Los resultados observados al diferenciar los estilos de aprendizaje en función del género son dispares (Esguerra \& Guerrero, 2009; Acevedo et al., 2015). Algunos estudios como el de Bahamón et al. (2013), GilMadrona et al. (2007), Blasco, et al. (2011) o Villalba (2015), han apreciado que el género femenino tiene predominancia en el estilo reflexivo. Esto conllevaría mayor capacidad para trabajar bajo presión y forma consciente, mayor capacidad receptiva y de análisis. Aunque este estilo se relaciona con dificultades para aprender cuando no se poseen estímulos/información suficiente, o cuando se deben desarrollar contenidos de manera superficial (Acevedo et al., 2015; González et al., 2015).

En el caso del género masculino, Gil-Madrona et al. (2007) han observado a predominancia por un estilo teórico. Así, los estudiantes serían más capaces de emplear la abstracción para reflexionar y desarrollar conclusiones, tanto a nivel metódico como lógico, intentando alcanzar nuevos aprendizajes de forma objetiva, crítica, estructurada y plani- ficada (Bahamón et al., 2013). Con todo ello, se percibe también la influencia de otras variables no controladas como la idiosincrasia propia de cada país, cultura, familia, sistema educativo o área de conocimiento. En este sentido, y en el caso de las carreras propias de Educación Física y Deporte, sería interesante profundizar incluso sobre los estilos de aprendizaje en función de la cantidad y el tipo de práctica realizada. Quizás, estas variables contribuyesen a entender las diferencias de género observadas en este trabajo (Delgado Noguera \& Zurita, 2003).

\section{Estilos de aprendizaje y edad}

Se apreció que los estudiantes menores de 21 años tienden a poseer una predominancia en el estilo activo, a diferencia de sus compañeros de mayor edad, que mostraron combinación de estilos. Estos resultados concuerdan con los observados al analizar la relación entre estilos de aprendizaje y curso, sugiriendo la adaptación del aprendizaje a medida que se progresa en la adquisición de nuevos conocimientos durante la vida universitaria. No obstante, estos resultados difieren de otros estudios en los que se ha observado que los estudiantes menores de 21 poseen un estilo reflexivo (Villalba, 2015), o en los que se apreciaron resultados dispares (Esguerra \& Guerrero, 2009). Estos datos, unidos a que no se observase una relación significativa entre ambas variables en este trabajo, develan que la edad (entendida como edad cronológica), no sería una variable determinante sobre el estilo de aprendizaje de los estudiantes.

De este modo se sugiere que el estilo de aprendizaje estaría mayormente influenciado por los procedimientos de adaptación funcional del estudiante a los procesos de enseñanza recibidos, en función de sus características y condicionantes concretos. Bajo este enfoque se resalta la necesidad de considerar el proceso de formación inicial docente como un proceso dinámico multivariable (Carreiro da Costa et al., 2016). Se trata de un proceso en el que tanto directivos, profesores, como encargados de cualquier acto pedagógico relacionado con el estudiante, poseen una herramienta fundamental para el logro de aprendizajes significativos.

\section{Estilos de aprendizaje y rendimiento académico}

La predominancia de un estilo pragmático o combinado se relacionó con resultados superiores de rendimiento académico. Estos hallazgos difieren de los observados por Esguerra \& Guerrero (2009), quienes relacionaron el estilo reflexivo con un rendimiento académico superior. Igualmente, los resultados de este trabajo se diferencian de los observados por Ossa \& Lagos (2013), que apreciaron la necesidad de una predominancia en los estilos teórico o reflexivo, para demostrar un rendimiento superior. No obstante, los estudiantes con un estilo activo en este trabajo demostraron un rendimiento significativamente inferior que los estudiantes con un estilo pragmático o combinado. Ello denota la relación entre el estilo activo y resultados académicos inferiores, como en otros estudios (Camarero et al., 2000; Esguerro \& Guerrero, 2009).

En cualquier caso, los hallazgos observados en este trabajo deben ser interpretados con cautela. Como se ha indicado en varias ocasiones, parece ser que el estilo de aprendizaje supone un complejo dinámico que se ve influenciado por multitud de factores (personales, sociales, culturales...). Por este motivo, afirmar que un estilo pragmático o combinado estaría relacionado con rendimientos académicos superiores sería arriesgado. Sin embargo, sí puede afirmarse que estos estilos están directamente relacionados con modelos de enseñanza-aprendizaje innovadores, así como con aprendizajes significativos (Wilkinson et al., 2014). Con todo ello, son necesarios más estudios que analicen la relación entre los métodos de enseñanza utilizados, los estilos de aprendizaje y los resultados académicos. Solo de este modo se podrá profundizar en este constructo tan complejo.

\section{Estilos de aprendizaje y área de conocimiento}

Si bien es cierto que se observó cierta similitud en los hallazgos observados en este trabajo y aquellos apreciados en otros estudios, en estudiantes de diferentes áreas de conocimiento (Acevedo et al., 2015; Gil-Madrona et al., 2007; González et al., 2015; Salas, 2014; Villalba, 
2015); los resultados en relación a la predominancia de los estilos de aprendizaje y las diferencias de género observadas, sugieren la particularidad de la muestra y su contexto (estudiantes de Educación Física, en Chile, en una universidad católica regional del sur, en un contexto concreto).

Además, cada perfil laboral de un titulado universitario, requiere de una serie de competencias específicas acordes a las funciones y propósitos de la profesión, e igualmente, una serie de habilidades propias del área de conocimiento en desarrollo. Por ejemplo, un especialista de la salud como un profesional de la Enfermería o la Terapia ocupacional, requierirá de una formación acorde a las necesidades físicas y también situación psicológica de sus pacientes. De igual modo, el profesional de Educación Física y Deporte deberá poseer un perfil adecuado al área, adaptado a las necesidades físicas y psicológicas, pero también sociales, culturales y económicas actuales de la población (López Pastor et al., 2016; Martins, Torres, Cardoso, Costa, \& Honório, 2015). Por este motivo, se intuye que el hecho de observar una predominancia del estilo combinado en los encuestados de este trabajo, podría estar relacionada también con un perfil multifacético adaptable, y funcional, del profesional de Educación Física y Deporte. Esto también explicaría el porcentaje superior de estudiantes con un estilo activo (con características propias del perfil, como ser: animador, espontáneo, arriesgado, creativo...), a diferencia de otros trabajos.

En definitiva, se resalta la necesidad de atender no solamente a la relación entre formación inicial docente y estilo de aprendizaje, sino también, a la relación existente entre las cualidades y capacidades individuales de los estudiantes, y sus necesidades de formación (implícitas en el perfil profesional propio).

\section{Conclusiones}

Se puede afirmar que los estudiantes de Pedagogía en Educación Física de la Universidad Católica de Temuco (Chile), poseen la predominancia de un estilo de aprendizaje combinado. Estos hallazgos se diferencian de otros estudios en los que se apreció la predominancia de un estilo reflexivo. El curso y la edad no se relacionan directamente con los estilos de aprendizaje de los estudiantes encuestados, aunque se observa una tendencia hacia la combinación de estilos con el aumento de curso y edad. Este hallazgo resalta la importancia de atender a los procesos de formación inicial docente desde una perspectiva flexible y adaptada al contexto sociocultural y educativo de los estudiantes, y a las particularidades del área de conocimiento profesional. Por otro lado, el género resultó ser una variable determinante sobre los estilos de aprendizaje, mostrando el género femenino una predominancia en el estilo activo. Finalmente, el estilo activo se relacionó con resultados académicos inferiores, siendo los estudiantes con estilos pragmático y combinado los que mejores resultados demostraron. Con ello, se percibe la necesidad de realizar nuevos trabajos en los que se analice la relación entre los estilos de aprendizaje y el rendimiento académico, e igualmente entre los estilos y el perfil profesional, principalmente desde una perspectiva de género.

\section{Referencias}

Acevedo, D., Cavadla, S., \& Alvis, A. (2015). Estilos de Aprendizaje de los Estudiantes de la Facultad de Ingeniería de la Universidad de Cartagena (Colombia). Formación Universitaria, 8 (4), 15-22.

Alonso, C.M. (1991). Estilos de aprendizaje: Análisis y diagnóstico en estudiantes universitarios. Tesis doctoral. Facultad de Filosofía y Ciencias de La EducaciónUniversidad Complutense de Madrid.

Bahamón, M., Vianchá, M., Alarcón, L., \& Bohórquez, C. (2012). Estilos y estrategias de aprendizaje relacionados con el logro académico en estudiantes universitarios. Pensamiento Psicológico, 9(1), 115-12.

Blasco, J.E., Romero, C., Mengual, S., Fernández-Revelles, A.B., Delgado, M.A. y Vega, L. (2011). Estilo de aprendizaje de los estudiantes de magisterio de educación física y de ciencias del deporte de las universidades de Granada y Alicante. Cultura y educación, 23, 371-383

Camarero, F., Buey, F., \& Herrero, J. (2000). Estilos y estrategias de aprendizaje en estudiantes universitarios. Psicothema, 12(4), 615622.

Carreiro da Costa, F., González Valeiro, M.A. \& González Villalobos, M.T. (2016). Innovación en la formación del profesorado de educación física. Retos, 29, 251-257.

Catalán, X., \& Santelices, M. (2014). Rendimiento Académico de estudiantes de distinto nivel socioeconómico en universidades: El caso de la Pontificia Universidad Católica de Chile. Calidad de Educación, 3, 22-52.

Cocca, A., Mayorga-Vega, D., \& Viciana, J. (2013). Relación entre niveles de actividad física y placer de ser activos en estudiantes universitarios. RIPED - Revista Iberoamericana de Psicología del Ejercicio y el Deporte, 8(2), 359-372.

Coloma, C., Manrique, L., Revilla, Diana, \& Tafur, R. (2008). Estudio descriptivo de los estilos de aprendizaje de docentes universitarios. Revista Estilos de aprendizaje, 1(1), 124-142.

Cordero, E., Lizano, C., Ortiz, A., \& Arias, F. (2015). Relationship between learning style and academic performance in students of the pharmacy program at Universidad de Costa Rica. Revista Digital de Investigación en Docencia Universitaria, 0 (2), 49-63.

Loret de Mola, J. (2008). Los estilos de aprendizaje de Honey - Alonso y el rendimiento académico en las áreas de formación general y formación profesional básica de los estudiantes del instituto superior pedagógico privado «Nuestra Señora de Guadalupe» de la provincia de Huancayo. Revista Estilos de Aprendizaje, 1(1), 201213.

Delgado-, M. A., \& Zurita, F. (2003). Estudio de las teorías implícitas de la educación física en la formación inicial de los maestros en las diferentes especialidades de la facultad de ciencias de la educación de la universidad de granada. Diferencias en función del género. Retos. Nuevas tendencias en Educación Física, Deporte y Recreación, 5, 27-38.

De Moya, Ma del Valle; Hernández, J. R., Hernández, J.A., \& Cózar, R.(2011). Análisis de los estilos de aprendizaje y las TIC en la formación personal del alumnado universitario a traves del cuestionario REATIC. Revista de Investigación Educativa, 29 (1), 137156.

Edel, R. (2003). Factores asociados al rendimiento escolar. Revista Iberoamericana de Educación, 1(2), 1-15.

Esguerrra, G, \& Guerrero, P. (2009). Estilos de aprendizaje y rendimiento académico en estudiantes de Psicología. Perspectivas en Psicología, 6(1), 97-109.

Garbanzo, G (2007). Factores asociados al rendimiento académico en estudiantes universitarios, una reflexión desde la calidad de la educación superior pública. Revista Educación, 31(1), 43-63.

García, G, \& De León, B. (2014). Estilos de Aprendizaje. Una revisión. Revista electronica de la divulgación de la investigación, 6, 1-8.

Gil-Madrona, P. (2004). Estilos de aprendizaje y Educación Física. En Alonso, C. y Gallego, D. (Eds.) I Congreso Internacional de Estilos de Aprendizaje. Madrid: UNED

Gil-Madrona, P., Contreras, O. P., Isabel, G., González, S., García, L., De Moya, M. d., \& López,A. (2007). Estilos de aprendizaje de los estudiantes de magisterio: Especial. Profesorado, Revista de $\mathrm{Cu}$ rrículum y formación de profesorado, 12(2), 1-19.

Gómez, P., Pérez, C., Parra, P., Ortiz, L., Matus, O., Mccoll, P., \&Mayer, A. (2015). Relación entre bienestar y el rendimiento académico en alumnos de primer año de medicina. Revista Médica de Chile, 143(7), 930-937.

Gómez-Mármol, A., \& Valero, A. (2015). Gestión del tiempo en las clases de educación física: estrategias para la mejora de los procesos de enseñanza. Journal of Sport and Health Research. 7(2),73-80.

González, E., Valenzuela, G, \& González,A. (2015). Diferencias significativas de los estilos de aprendizaje con las características del estudiante universitario en México. Revista de Estilos de Aprendi- 
zaje, 8 (15), 201-221.

Isaza, L. (2014). Estilos de Aprendizaje: una apuesta por el desempeño académico de los estudiantes en la Educación Superior. Revista Encuentros, Universidad Autónoma del Caribe, 12(2), 25-34.

López Pastor, V. (2016). Los retos de la Educación Física en el Siglo XXI. Retos, (29), 182-187.

Madrid-López, P.D., Prieto-Ayuso, A., Samalot-Rivera, A., \& GilMadrona, P. (2016). Evaluación de una propuesta extraescolar de conductas apropiadas en educación física y deportiva. Retos. Nuevas tendencias en Educación Física, Deporte y Recreación 30, 3642.

Margalef, G (2014). Evaluación formativa de los aprendizajes en el contexto universitario: Resistencias y paradojas del profesorado. Educación XX1,17(2), 1-12.

Martínez, R. (2007). La investigación en la práctica educativa: Guía metodológica de investigación para el diagnóstico y evaluación en los centros docentes. Madrid: Centro de Investigación y Documentación Educativa (CIDE).

Martins, J., Torres, B., Cardoso, J., Costa, A.M., \& Honório, S. (2015). Influence of sociological aspects on the level of physical activity in physical education students. J. JOURNAL OF HUMAN SPORT \& EXERCISE, 10(3), 815-826.

Maureira, F. (2013). Validez y confiabilidad del CHAE en estudiantes de educación física. Educación Física Chile, 8-15.

Maureira, F. (2015). CHAEA-36: Adaptación del cuestionario HoneyAlonso de estilos de aprendizajes para estudiantes de educación física de Chile. Revista Electrónica de Psicología Iztacala, 18(3), 1133-1152.

Monroy, F., \& Hernandez, F. (2014). Factores que influyen en los enfoques de aprendizaje universitario. Una revisión sistemática. Revista Educación XXI, 17 (2), 105-124. doi:10.5944/ educxx1.17.2.11481

Nuñez, J., \& Reyes, C. (2014). La evaluación del aprendizaje de estudiantes: validación española del Assessment Experience Questionnaire (AEQ). ESTUDIOS SOBRE EDUCACIÓN, 26, 63-77.

Ortiz, A., \& Canto, P. (2013). Estilos de aprendizaje y rendimiento académico en estudiantes de ingeniería en México. Revista de estilos de aprendizaje, 6(11), 160-177.
Ossa, C., \& Lagos, N. (2013). Estilos de Aprendizaje y rendimiento académico en estudiantes de Pedagogía de Educación General Básica (Primaria de una universidad pública en Chile). Revista Estilos de Aprendizaje, 6(11), 178-189.

Ramírez, P., \& Fuentes, C. (2013). Felicidad y Rendimiento Académico: Efecto Moderador de Felicidad sobre indicadores de Selección y RendimientoAcadémico deAlumnos de Ingenieria Comercial. Formación Pedagógica, 6(3), 21-30.

Salas, J. (2014). Estilos de aprendizaje en estudiantes de la Escuela de Ciencias del Movimiento Humano y Calidad de Vida, Universidad Nacional, Costa Rica. Revista electronica EDUCARE, 23(3), 159171.

Santizo, J., García, L., \& Gallego, D. (2008). Dos métodos para la identificación de diferencias de estilos de aprendizaje entre estudios donde se ha aplicado el CHAEA. Revista Estilos de Aprendizaje, 1(1), 28-42.

Urval, R., Kamath, A., Ullal, S., Shenoy, A., Shenoy, N., \& Udupa, L. (2014). Assessment of learning styles of undergraduate medical students using the VARK questionnaire and the influence of sex and academic performance. Advances in Physiology Education, 38(3), 216-220.

Vélez, A., \& Roa, C. (2005). Factores asociados al rendimiento académico en estudiantes de medicina. Educación Médica, 8(2), 74-82.

Villalba, A. (2015). Estilos de aprendizaje en alumnos universitarios de profesorado en Biología y licenciatura en Biodiversidad. Revista Estilos de Aprendizaje, 8(16), 79-100.

Villalta, M. (2010). Factores de resiliencia asociados al rendimiento académico en estudiantes de contextos de alta vulnerabilidad social. Revista de Pedagogía, 31(88), 159-188.

Wilkinson, T., Boohan, M., \& Stevenson, M. (2014). Does learning style influence academic performance in different forms of assessment? Journal of Anatomy, 224(3), 304-308.

Zagalaz Sánchez, M.L., Cachón Zagalaz, J., \& Lara Sánchez,A.J.(2014). Fundamentos de la programación de Educación Física en primaria. Madrid: Síntesis. Zamorano García, M.; Gil Madrona, P.; PrietoAyuso, A. y Zamorano García, D. (201x). Generated Emotions by Various Types of Games in Physical Education. Revista Internacional de Medicina y Ciencias de la Actividad Física y el Deporte vol. x (x) pp.xx Pendiente de publicación / In press

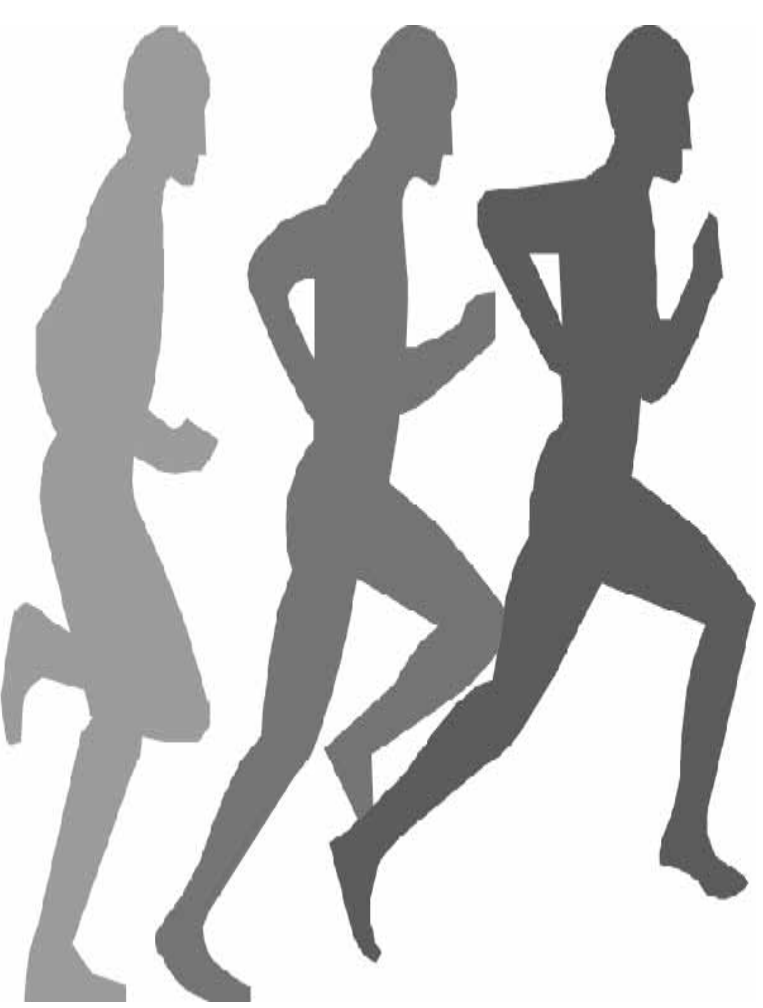

